\title{
Prevalencia de estrés en escolares de Tuxtla Gutiérrez Chiapas, México y factores familiares asociados
}

\section{Prevalence of stress among schoolchildren, and associated family factors, in Tuxtla Gutierrez, Chiapas, Mexico.}

Fecha de recepción: 25/08/2014

Fecha de aceptación: 22/12/2014

\author{
Alfredo Briones Aranda ${ }^{1}$, Victor Vega Villa ${ }^{1}$, \\ Gonzalo López Aguirre ${ }^{1}$, Manuela Castellanos Pérez ${ }^{l}$ y \\ José Eduardo Suárez Santiago ${ }^{2}$ \\ Profesores investigadores Facultad de Medicina Humana. \\ Universidad Autónoma de Chiapas (UNACH), Tuxtla Gutiérrez Chiapas. México. \\ 2Estudiante de doctorado de la Escuela Superior de Medicina del \\ Instituto Politécnico Nacional. México.
}

\section{resumen/abstract:}

En la actualidad, el estrés infantil representa un factor ascendente y silencioso que influye negativamente en la salud de los niños. En la presente investigación se estudió la prevalencia de estrés en una muestra representativa de 255 escolares entre 9 y 10 años de edad (132 niñas y 123 niños), que cursaban el cuarto año de primaria en la Ciudad Tuxtla Gutiérrez Chiapas, México. Se aplicó el Inventario de Estrés Infantil de Vega, posteriormente, en los padres de los niños con estrés marcado se les realizó una entrevista cerrada encaminada a detectar disfuncionalidad familiar. Se encontró un alto porcentaje de estrés marcado (43.1\%), con predominio en las niñas (27\%), en la entrevista de los padres fue comúnmente referida la disolución conyugal. Las diferencias en la percepción del estrés influenciada por el sexo podría explicar el predomino del estrés en las niñas, mientras que la disolución conyugal fue acorde con la estadística sociodemográfica de la región y encuadra con los factores generadores de estrés. La anterior información debería ser tomada en cuenta para el diseño de las políticas estatales de salud, a fin de frenar el impacto negativo del estrés en el perfil de crecimiento y desarrollo de la sociedad.

Nowadays childhood stress represents an increasingly important factor that silently and negatively influences the health of children. The current contribution analyzes the presence of stress in a representative sample of 255 primary school children between 9 and 10 years old (132 girls and 123 boys) that were in the fourth grade in Tuxtla Gutierrez, Chiapas, Mexico. Stress was detected in these children by employing the Inventory of Childhood Stress by Vega. Afterwards, a closed interview was carried out with the parents of stressed children to explore the possibility of a dysfunctional family. In a large percentage (43.1\%) of children, a notably high level of stress was found. Among stressed children, the majority of were girls (27\%), and for both genders separation of the parents was common. Marital dissolution produces well known stress factors. The percentage of marital dissolution among parents in the study was in accordance with demographic statistics. The greater percentage of girls experiencing stress could be explained by the differences in perception of stress influenced by gender. These facts should be taken into account by state health authorities when designing policies for school children, with the aim of diminishing the negative impact of stress in the growth and development of society.

\section{palabras clave/keywords:}

Estrés infantil, disolución conyugal, disfuncionalidad familiar, Tuxtla Gutiérrez Chiapas

Childhood stress, marital dissolution, dysfunctional family, Tuxtla Gutierrez, Chiapas

correspondencia: Dr. Alfredo Briones Aranda: alfred725@hotmail.com 


\section{Introducción}

Uno de los costos inherentes al progreso de nuestra sociedad actual es el desarrollo de nuevas patologías ligada al estrés. El estrés tiene infinidad de matices como los colores de una pintura, tan solo la clasificación del estrés puede girar en torno a la temporalidad y tipos de factores que lo generan, así como a los distintos grupos etarios que lo padecen (Del Giudice, 2014; Sävendahl, 2012).

Los niños interactúan activamente con el ambiente y son receptores dinámicos de las fuerzas ambientales. El estrés se presenta en los niños cuando un factor físico o psicológico ejerce una presión importante en su habilidad para adaptarse y conservar la homeostasis; de acuerdo con el tiempo de exposición a los factores estresantes, los efectos en los niños pueden ser agudos o impredecibles, crónicos o previsibles, incluida una tercera categoría denominada estresores neutros, construidos por problemas o dificultades cotidianas (Afifi, Boman, Fleisher y Sareen, 2009; Sävendahl, 2012; Verduzco, Gómez y Durán, 2004).

En México se carece de estudios relacionados con la prevalencia del estrés infantil. Sin embargo, existen investigaciones puntuales que pudieran aportar bases para construir un panorama general; por ejemplo, los problemas de salud mental que tienen mayor demanda en la consulta externa de las instituciones psiquiátricas públicas en México son los relacionados con el estrés, como los trastornos depresivos, disociales, al igual que los problemas de aprendizaje (Secretaría de Salud, 2001). Recientemente Benjet et al. (2009), reportaron que el $68 \%$ de adolescentes, en una muestra estudiada en la ciudad de México, experimentó al menos un tipo de adversidad crónica durante su infancia.

Los estresores infantiles están fuertemente asociados es un inicio al núcleo familiar y las relaciones de apego; con la entrada del niño a la escuela, los estresores en la infancia pueden situarse en el contexto escolar y en la relación con sus compañeros (Oros y Vogel, 2005). En el estudio con adolescentes mexicanos se encontró que la adversidad más frecuente fue la económica, seguida de la violencia doméstica. También se reportó que los niños presentaron mayor abandono que la niñas y el abuso sexual fue más frecuente en estas últimas (Benjet et al., 2009).

El abandono emocional en la infancia se ha relacionado significativamente con la aparición de trastornos de ansiedad que contribuyen también a aumentar la gravedad de las psicopatologías en la vida adulta (Hovens et al., 2010; Sareen, Fleisher, Cox, Hassard y Stein, 2005). Igualmente, el abuso sexual en etapas tempranas de la vida se ha asociado con la prevalencia de trastornos de ansiedad, específicamente con el trastorno de estrés postraumático (Afifi et al., 2008; Caspi et al., 2008; Gibb, Chelminski, y Zimmerman, 2007; Green et al., 2010; Hovens et al., 2010; Kessler et al., 2010; Khoury, Tang, Bradley, Cubells, y Ressler, 2010; Lang, Stein, Kennedy y Foy, 2004; McLaughlin et al., 2010; Sareen et al., 2005; Wingenfeld et al, 2011).

La presencia de estrés durante el desarrollo del niño puede causar daños neurobiológicos y neuroendocrinos que podrían mantenerse durante el resto de la vida del sujeto y causar serias alteraciones en las áreas conductuales, emocionales, sociales y cognitivas (Becker y Grilo, 2011; Bremne y Vermetten, 2001; Roy, 2002)

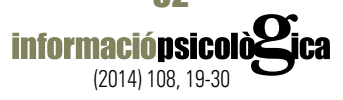


La importancia del estudio del estrés en la infancia radica en que esta condición pudiese servir como un elemento predictor para el desarrollo de diversos trastornos mentales en la adultez (Hovens et al., 2010; McLaughlin et al., 2010; Molnar, Buka y Kessler, 2001; Roy, 2002). Por ejemplo, la presencia de estrés en la infancia se asocia con un 20-60\% más de riesgo para desarrollar alguna psicopatología y representa casi $45 \%$ de la mayoría de los trastornos de inicio en la infancia y hasta $32 \%$ de los trastornos mentales en la vida adulta (Afifi et al., 2008; Green et al., 2010).

Por todo lo anterior, el propósito principal de la presente investigación fue estudiar la prevalencia de estrés en una muestra significativa de niños escolares tuxtlecos que cursaban el cuarto año de primaria, así como tratar de conocer los principales factores familiares implicados.

\section{Método}

\section{Participantes}

Los escolares participantes en este estudio fueron niños, y sus respectivos padres o tutores, habitantes de la ciudad de Tuxtla Gutiérrez, Chiapas, México que tenían entre 9 y 10 años de edad y se encontraban inscritos en alguna escuela primaria pública.

\section{Muestra de escolares}

Para la integración de la muestra de estudio se consultó el censo de población y vivienda 2010, realizado por el Instituto Nacional de Estadística y Geografía (INEGI), el cual arrojó un total de 19740 de niños entre 9 y 10 años de edad.

Posteriormente se procedió a calcular la muestra con un error estimado de $5 \%$ y un nivel de confianza del $95 \%$, cuyo resultado fue un número estimado de 250 niños, por lo que se decidió hacer un muestreo por conglomerados dividiendo a la ciudad en 5 zonas: centro, norte, sur, oriente y poniente. Por conveniencia y facilidad se determinó trabajar con una de las escuelas primarias ubicada en cada zona y en donde la elección sería al azar.

La muestra estuvo representada por 123 niños y 132 niñas que cumplieron los siguientes criterios de inclusión: Niños escolares tuxtlecos que cursaran el cuarto año de primaria, de entre 9 y 10 años de edad, sin padecimiento de algún trastorno neuropsicológico. El estudio fue desarrollado de julio a diciembre de 2013 en los escolares que contaban con un consentimiento firmado del padre o tutor, y se respaldó con la autorización del Comité de Ética de la Facultad de Medicina Humana de la Universidad Autónoma de Chiapas.

\section{Instrumentos de evaluación}

Para evaluar los niveles de estrés de los niños se utilizó el inventario de estrés infantil (IEI), que consta de 53 ítems con tres subescalas: familiar con 19, escolar con 13 y social con 19 ítems. Las opciones de respuestas fueron: muy contento, poco contento, nada contento; nada nervioso, poco nervioso y muy nervioso. Se calificó de uno a seis puntos, otorgando un punto a muy contento y seis puntos a muy nervioso. La puntuación mínima fue de 52 y la máxima de 312, a mayor puntuación mayor estrés (Vega, González, Anguiano, Nava y Soria, 2009). 
También se aplicó una entrevista cerrada a los padres o tutores, enfocada a conocer factores puntuales de disfuncionalidad familiar que pudieran influir en la generación de estrés en los niños, la cual se realizó de manera personalizada y tuvo una duración aproximada de 10 minutos por padre o tutor.

\section{Procedimiento}

Después de seleccionar la muestra de los alumnos se procedió a la administración del IEI a los seleccionados de las cinco escuelas primarias estudiadas. La aplicación de los inventarios se llevó a cabo en una sola sesión de aproximadamente $30 \mathrm{~min}$. Estas aplicaciones se realizaron de manera grupal en el salón de clases de cada escuela, por tres personas que recibieron entrenamiento previo.

Una vez detectados los escolares con el mayor grado de estrés, se concertó una cita con el padre o tutor para informarle acerca de los resultados que el niño obtuvo en la prueba psicológica y posteriormente se realizó la entrevista cerrada.

Se realizó un análisis descriptivo de los datos mediante el programa SPSS 19 tomando en cuenta el número de casos y el porcentaje del grado de estrés que los escolares presentaron de acuerdo con la zona de estudio y el sexo, asimismo, se analizaron las puntuaciones de las distintas áreas de la prueba en los escolares que presentaron estrés leve y marcado. Finalmente se tabularon las principales respuestas de los padres o tutores de niños con estrés marcado.

\section{Resultados}

Los niños tuvieron el mayor número de casos sin datos de estrés, en dos de las cinco zonas de estudio (II y III). Contrariamente a los niños, el grupo de las niñas presentó el mayor porcentaje de estrés marcado, el cual predominó en la totalidad de las zonas de estudiadas (tabla 1); mientras que el número y porcentaje de casos fue muy homogéneo para el estrés leve, cuando se comparó a los niños respecto de las niñas, en la mayoría de las zonas de estudio.

El alto porcentaje de estrés marcado que presentaron las niñas tendió a duplicarse o triplicarse en proporción cuando se comparó con respecto del grupo de las niñas que no manifestaron datos de estrés.

Por otro lado, los niveles de estrés en el grupo de los niños se caracterizaron por una alta proporción de estrés marcado pero, a diferencia de las niñas, únicamente se pudo observar esta proporción alta en tres de las cinco zonas estudiadas (I, IV y V). Por lo anterior, el grupo de las niñas presentó el mayor número y porcentaje de casos con estrés marcado, en el total de la población estudiada. 


\begin{tabular}{|c|c|c|c|c|c|c|}
\hline \multicolumn{7}{|c|}{ Tabla1. Estrés en escolares con relación al sexo y a la zona de estudio. } \\
\hline \multirow[b]{3}{*}{$\begin{array}{l}\text { Zonas de } \\
\text { estudio }\end{array}$} & \multicolumn{6}{|c|}{ Niveles de estrés } \\
\hline & \multicolumn{2}{|c|}{ S. E. } & \multicolumn{2}{|c|}{ E. L. } & \multicolumn{2}{|c|}{ E. M. } \\
\hline & $\begin{array}{c}\mathrm{V} \\
\mathrm{n}, \%\end{array}$ & $\begin{array}{c}M \\
n, \%\end{array}$ & $\begin{array}{c}V \\
n, \%\end{array}$ & $\begin{array}{c}M \\
n, \%\end{array}$ & $\begin{array}{c}V \\
n, \%\end{array}$ & $\begin{array}{c}M \\
n, \%\end{array}$ \\
\hline I & $6,28.0$ & $5,25.0$ & $8,36.0$ & $7,35.0$ & $8,36.0$ & $8,40.0$ \\
\hline$\|$ & $23,60.5$ & $10,27.0$ & $7,18.4$ & $10,27.0$ & $8,21.0$ & $17,46.0$ \\
\hline III & $12,42.8$ & $8,18.2$ & $9,32.0$ & $13,29.0$ & $7,25.0$ & $23,52.3$ \\
\hline IV & $8,40.0$ & $1,5.0$ & $1,5.0$ & $5,25.0$ & $11,55.0$ & $14,70.0$ \\
\hline V & $6,40.0$ & $2,18.0$ & $2,13.4$ & $2,18.2$ & $7,46.6$ & 7, 63.6 \\
\hline $\begin{array}{l}\text { El cuadro } \\
\text { grado de } \\
\text { números }\end{array}$ & $\begin{array}{l}\text { sa el Núr } \\
\text { (S. E. Sir } \\
\text { cados de }\end{array}$ & $\begin{array}{l}\text { I } 0 \text { el por } \\
\text { s, E.L. est } \\
\text { a mavor }\end{array}$ & $\begin{array}{l}\text { del tota } \\
\text { M. Est } \\
\text { de caso }\end{array}$ & $\begin{array}{l}\text { os (V) o nir } \\
\text { cado), en o } \\
\text { rados de a }\end{array}$ & $\begin{array}{l}\text { estudiadas } \\
\text { a de las zor } \\
\text { al sexo y a }\end{array}$ & $\begin{array}{l}\text { na, segú } \\
\text { udiadas. } \\
\text { a. }\end{array}$ \\
\hline
\end{tabular}

Cuando se analizó qué áreas podrían estar generando mayor impacto sobre los niveles de estrés leve y marcado en los escolares, se encontró que la mayoría de infantes que presentaron una alta puntuación en más del $60 \%$ de las preguntas correspondió, en primer término al área familiar y en segundo lugar al área escolar (ver tabla 2).

\begin{tabular}{|c|c|c|c|c|c|c|c|c|c|c|c|c|}
\hline \multirow{3}{*}{$\begin{array}{l}\text { Zonas de } \\
\text { estudio }\end{array}$} & \multicolumn{6}{|c|}{ Estrés leve } & \multicolumn{6}{|c|}{ Estrés marcado } \\
\hline & \multicolumn{2}{|c|}{ Familiar } & \multicolumn{2}{|c|}{ Escolar } & \multicolumn{2}{|c|}{ Social } & \multicolumn{2}{|c|}{ Familiar } & \multicolumn{2}{|c|}{ Escolar } & \multicolumn{2}{|c|}{ Social } \\
\hline & V & M & V & M & V & $M$ & V & $M$ & V & $M$ & V & M \\
\hline I & 5 & 6 & 3 & 2 & & & 7 & 8 & 1 & & & \\
\hline$\|$ & 4 & 6 & 2 & 3 & 1 & 1 & 6 & 14 & 1 & 3 & 1 & \\
\hline III & 6 & 10 & 1 & 3 & 2 & & 4 & 21 & 2 & 2 & 1 & \\
\hline IV & 1 & 5 & & & & & 10 & 14 & 1 & & & \\
\hline V & & & 2 & 2 & & & 5 & 7 & 2 & & & \\
\hline
\end{tabular}




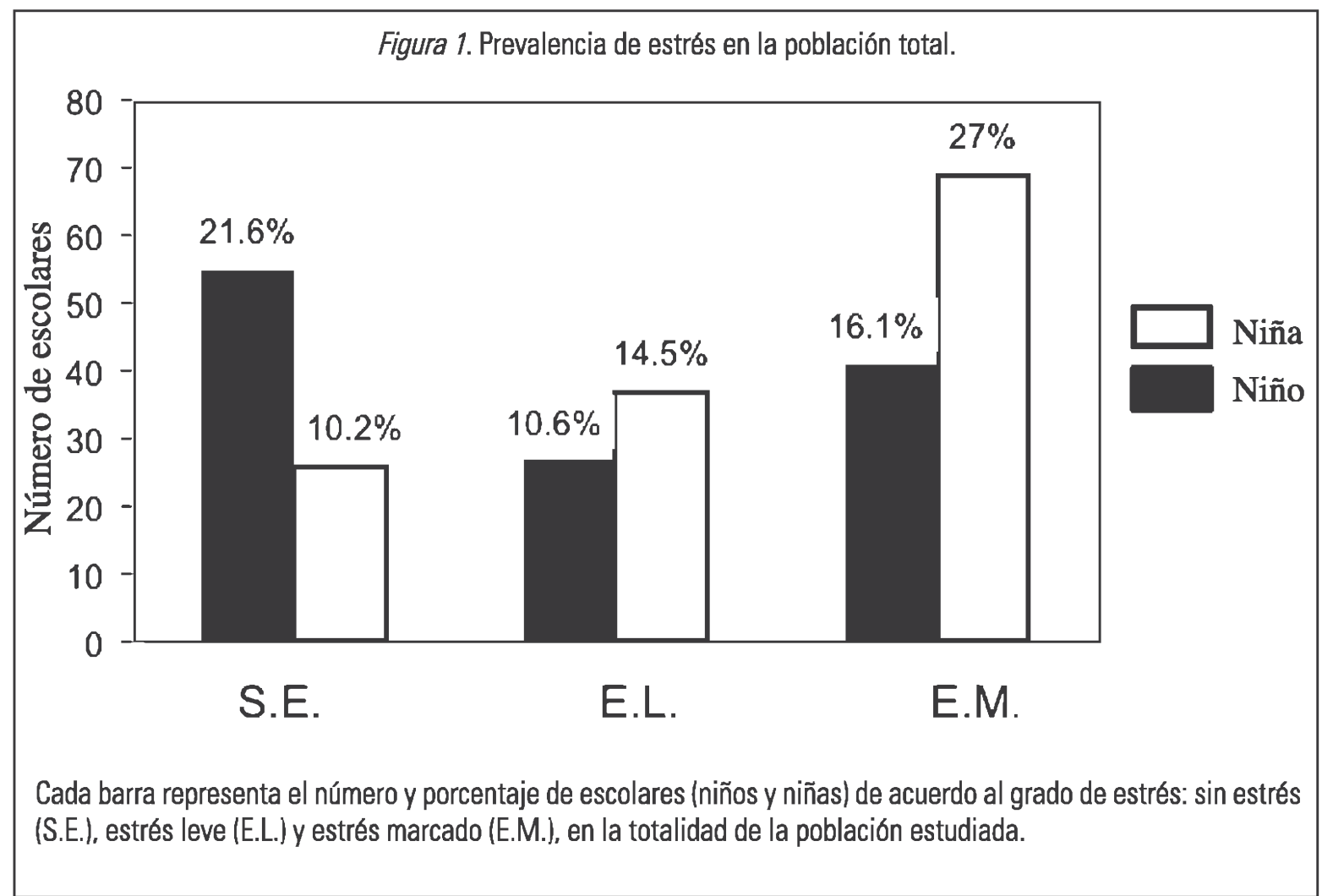

En el análisis del IEI de forma gráfica, de acuerdo con el sexo, en la totalidad de la población estudiada (Figura 1) se pudo constatar que en los niños el mayor porcentaje no presentó estrés (21.6\%), el segundo lugar lo ocupó el estrés leve con $16.1 \%$ y el tercer lugar el estrés marcado con un $10.6 \%$. En contraste, en el grupo de las niñas un alto porcentaje presentó estrés marcado (27.0\%), seguido de estrés leve (14.1\%) y sin estrés (10.2\%).

\begin{tabular}{|lcc|}
\hline \multicolumn{2}{|c|}{ Tabla 3. Principales respuestas en entrevistas de los padres o tutores de escolares con estrés marcado. } \\
\hline \multicolumn{1}{|c|}{ Respuestas } & Número de escolares & Porcentaje (\%) \\
\hline Padres divorciados o separados & 37 & 33.6 \\
Padres en proceso de divorcio o separación & 24 & 21.8 \\
$\begin{array}{l}\text { Padre o madre ausente en la casa por muchas horas o días } \\
\text { por situaciones laborales }\end{array}$ & 21 & 19.0 \\
Alguno de los padres o ambos con alguna adicción & 16 & 14.6 \\
Presencia de padrastro o madrastra en la familia & 12 & 11.0 \\
\hline
\end{tabular}


En la entrevista cerrada a los tutores de los escolares con estrés marcado se encontró que las principales respuestas se referían en primer lugar a la separación o proceso de divorcio de los padres; en segundo lugar, al descuido o abandono de los hijos por situaciones laborales de los padres; en tercer lugar, a la adicción de algunos de ellos; y en cuarto lugar, a la presencia de padrastro o madrastra en la familia (ver tabla 3 ).

\section{Discusión}

A manera de resumen, los resultados principales de este trabajo fueron los siguientes: en la totalidad de la población estudiada se detectó un alto porcentaje de estrés marcado $(43.1 \%)$. Esta alta prevalencia fue predominante en las niñas (27\%) en comparación con los niños (16.1\%), en donde la disolución conyugal de los padres es uno de los factores principales que podría estar relacionado.

La importancia de la presencia de estrés en los escolares radica en el impacto negativo que ejerce este factor sobre su salud y desarrollo (Sävendahl, 2012), especificamente sobre las esferas: biológica, psicológica y social. Por ejemplo, es bien conocido que durante el estrés existe una alta liberación de corticoesteroides (CE) en la sangre, cuya relevancia reside en su poder de interacción con diversos sistemas del organismo, entre los que se destaca el sistema inmunológico y el nerviosos central (SNC).

En el sistema inmune los CE pueden regular la capacidad fagocítica de los macrófagos, disminuir la síntesis de anticuerpos y ejercer poder antinflamatorio a través de la inhibición de la síntesis de las prostaglandinas (Elenkov et al., 1996; 1999). Lo anterior puede contribuir a la patogénesis de diversas enfermedades, desde simples resfriados comunes (Tasker et al., 2004; Turner, Rixon y Jessop, 2011) hasta enfermedades más complejas, como las autoinmunes (Espinosa et al., 2003; Mäenpää, Savolainen, Lehto y Belt, 2001; Straub y Härle, 2005).

En el SNC los CE pueden interaccionar en la regulación de multiples sistemas de neurotransmisión, entre los que se destaca al sistema noradrenérgico, serotoninérgico, GABAérgico, etc. (Briones et al. 2012; Höschl y Hajek, 2001; Leonard, 2006; Lightman, 2008). Casualmente estos mismos sistemas de neurotransmisión y dependiendo de la magnitud y el tiempo de exposición al estrés en los niños (Sävendahl, 2012), se han podido vincular con el desarrollo de diversos trastornos: depresivos, de ansiedad y problemas de aprendizaje (Ferro y Boyle, 2014; Lambert y Spinath, 2013; Talge, Neal, y Glover, 2007).

En un estudio realizado por Vega, Espindola, Nava, y Soria (2009), se reportó una alta incidencia de estrés en los escolares que cursaban el cuarto grado de primaria, más acentuado en las niñas que en los niños; llama la atención la gran similitud de estos resultados con los encontrados en la presente investigación. Sin embargo, la posible explicación de la tendencia al predominio del estrés en las niñas fue abordado de manera superficial en el estudio de Vega et al. (2009).

En concordancia con lo anterior, otro estudio de Loredo, Mejía, Jiménez, y Matus (2009), encontró que las niñas de primaria presentaron mayores niveles de estrés que los niños, 
cuya explicación giró en torno a la discrepancia de la percepción de los factores estresantes, idea que va acorde con lo reportado previamente en otro estudio, en donde se reafirma que las niñas perciben más los estresores relacionados con la pérdida afectiva de las personas cercanas, los problemas de salud de otros y las injusticias familiares. También, las niñas fueron las únicas que reconocieron estados de soledad (Oros y Vogel, 2005).

Es evidente que multiples factores confluyen en las diferencias de percepción ante los factores estresantes por parte de los niños, aunque es claro que existen áreas categóricas primordiales, como las diferencias en la maduración y desarrollo influenciadas por el factor genético y hormonal (Dosaev, Prakash y Livshits, 2014; Tharandt et al., 1981), evidencias que no deben pasar inadvertidas tanto en la vida cotidiana y como en los futuros estudios.

Por otra parte, en esta investigación se observó que los factores familiares pudieran estar asociados al alto porcentaje de escolares con estrés marcado, específicamente por la alta incidencia de disoluciones conyugales que predominó en las respuestas de los padres entrevistados que tenían hijos con estrés marcado. En relación con la veracidad de esto último, existen evidencias, tanto directas como indirectas, que pudieran reafirmar dicha correlación. Como ejemplo de evidencias directas, podríamos citar los conflictos generados en la relación de los padres, ya que es uno de los principales estresores que se destaca en la percepción de los niños, además de los estresores que sobresalen por su frecuencia de aparición en la infancia, como la ausencia paterna, la enfermedad o muerte de los padres, el divorcio, entre otros.

Al respecto, las estadísticas en América son alarmantes: el 40.0\% de los niños experimenta el divorcio de los padres, el $22.0 \%$ vive en pobreza, el $6.6 \%$ vive con un progenitor alcohólico. Si bien esos datos pertenecen a la década de los años noventa, puede sospecharse que en la actualidad aún son más desalentadores, sobre todo en el tipo de unión conyugal designado como unión libre, el cual tiene mayores probabilidades de disolución conyugal (Ojeda y González, 2008; Schmeer y Kroeger, 2011). Por citar un ejempo cercano, en la ciudad de Tuxtla Gutiérrez hubo 3185 nuevos casamientos y 685 divorcios en el año 2012 (estadística de nupcialidad; INEGI, 2012); esto quiere decir que por cada cuatro parejas que se casan, una se divorcia, sin tomar en cuenta que de las 172089 personas de 15 a 49 años en unión conyugal en el 2010, solo $117420(68.2 \%)$ estaban casadas por el régimen civil y el resto vivían en unión libre 54679 (31.8\%) (censo INEGI 2010), lo cual hace factible suponer que los conyuges que viven en unión libre también pueden separse pero son dificíles de tomar en cuenta para la estadística sociodemográfica, ya que las personas en esta condición no están obligadas a notificar este proceso e, incluso, en las personas casadas por el régimen civil, su disolución conyugal no necesariamente puede seguir un proceso legal de divorcio.

Es así como el número puntual de los diferentes tipos de disoluciones conyugales no se pueden determinar con exactitud. Sin embargo, al analizar el número de personas que viven divorciadas (5573) y separadas (14403) (censo INEGI 2010), se reafirma que el número de personas separadas es tres veces mayor al de las personas divorciadas, condición que refleja indirectamente el alto índice de disoluciones conyugales que no son reportadas.

En relación con las evidencias indirectas, en la entrevista de los padres o tutores de los niños con estrés marcado se encontró que un alto porcentaje de los padres estaban divorciados o en proceso de separación.

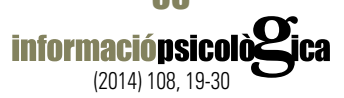


Dentro de las causas de la disolución conyugal existe una carencia de estudios actuales en México. No obstante, de manera puntual se ha evidenciado por estudios pequeños que el factor económico, seguido de las adicciones, se destacan entre las más importantes, información que tiene concordancia con el alto porcentaje $(48.0 \%)$ de la poblacion de Tuxtla Gutiérrez que se sufre algún grado de pobreza (censo INEGI 2010). A su vez, la Encuesta Nacional de Adicciones (2011) reveló que la dependencia al alcohol se duplicó entre 2002 y 2011, al pasar de 3.5 a 6.2\% en los hombres, y en las mujeres se triplicó pues subió de 0.6 a $2 \%$. Además que en esta misma encuesta se reportó el incremento constante y sostenido en la dependencia al alcohol, situación que se perfila como una de las adicciones que ocupa el primer lugar en la población adulta tuxtleca (Villatoro et al., 2011).

Retomando el análisis de la disolución conyugal es muy probable que esta condición esté influenciada sobre el estrés marcado de los niños, ya que la desintegración familiar conlleva a un aumento directo en el grado de estrés que sufren los hijos, por la incertidumbre del futuro y la violencia familiar que perciben a lo largo del proceso (Maganto, 2004; Benjet, Borges y Medina, 2010).

La violencia familiar implica cualquier acto deliberado de la fuerza física o el poder, ya sea en grado de amenaza o efectivo, de cuyo objetivo sea ejercer un dominio absoluto sobre cualquier integrante de la familia. Esta forma de violencia constituye un problema frecuente en las familias mexicanas. Nuestra cultura favorece una condición de falta de poder en la mujer que, en el ámbito de la pareja, la pone en riesgo de experimentar maltrato; dicha situación es también reproducida en las relaciones con los hijos, quienes a su vez, son maltratados por el padre o la madre. Este daño se produce al violar o invadir los espacios de quienes forman parte del grupo familiar del agresor para quitarles seguridad y poder, y mantenerlos generalmente en desequilibrio, con el objeto de vencer su resistencia y obtener su subyugación, es decir, lograr su control y dominio (Villatoro, Quiroz, Gutiérrez, Díaz y Amador, 2006). Mientras más espacios invade el victimario, mayor control ejercerá sobre sus víctimas.

En conclusión, el problema de la violencia familiar puede ser traducida en maltrato infantil, factor sumamente importante ya que la violencia se relaciona directamente con el grado de estrés; además es del conocimiento general que a mayor disfunción familiar, mayor violencia y consecuentemente mayor estrés (Benjet, et al., 2010). Un niño empieza a percibir estrés desde el momento que se generan discusiones entre sus padres al inicio del divorcio y hasta pasada la separación, por la nueva dinámica de vida, lo que hace que el nivel de estrés de los hijos de padres divorciados sea continuo y constante (Benjet, et al., 2010; Wolchik, Wilcox, Tein y Sandler, 2000).

La importancia del estudio del estrés infantil radica en la repercusión negativa que éste pudiera tener en la salud mental del adulto, ya que existe una relación significativa entre el grado de estrés que experimenta el niño y el desarrollo de psicopatologías en la edad adulta, como la depresión, la ansiedad y los trastornos cognitivos, principalmente en mujeres (Hovens et al., 2010; McLaughlin et al., 2010).

Por lo anterior es necesario y urgente continuar la investigación de la situación de estrés en la población infantil, condición que tambien debería tomarse en cuenta en el diseño y

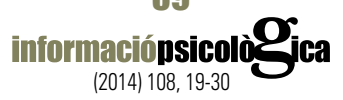


ejecución de estrategias gubernamentales en salud que coadyuven en la solución de este problema, así como de las consecuencias a corto y largo plazo.

Sin embargo, somos conscientes de que este primer acercamiento del estudio situacional del estrés infantil en la ciudad de Tuxtla Gutiérrez, sirvió para identificar otros factores etiológicos del estrés infantil no considerados en la presente investigación, así como su correspondiente correlación.

\section{Referencias}

Afifi, T. O., Boman, J., Fleisher, W., y Sareen, J. (2009). The relationship between child abuse, parental divorce, and lifetime mental disorders and suicidality in a nationally representative adult sample. Child Abuse Neglect, 33 (3), 139-147.

Afifi, T. O., Enns, M. W., Cox, B. J., Asmundson, G. J. G., Stein, M. B., y Sareen, J. (2008). Population attributable fractions of psychiatric disorders and suicide ideation and attempts associated with adverse childhood experiences. The American Journal of Public Health, 98, 946-952.

Becker, D. F., y Grilo, C. M. (2011). Childhood maltreatment in women with binge-eating disorder: Associations with psychiatric comorbidity, psychological functioning, and eating pathology. Eating and Weight Disorders, 16 (2), e113-120.

Benjet, C., Borges, G., y Medina-Mora, M. E. (2010). Chronic childhood adversity and onset of psychopathology during three life stages: childhood, adolescence and adulthood. Journal Psychiatry Research, 44 (11), 732-40.

Benjet, C., Borges, G., Medina-Mora, M. E., Zambrano, J., Cruz, C., y Méndez, E. (2009). Descriptive epidemiology of chronic childhood adversity in Mexican adolescents. Journal of Adolescent Health, 45 (5), 483-9.

Bremne, J. D., y Vermetten, E. (2001). Stress and development: Behavioral and biological consequences. Development and Psychopathology, 13 (3), 473-489.

Briones, A., Gagno, S., Martisova, E., Dobarro, M., Aisa, B., Solas, M., Tordera, R., y Ramírez, M. (2012). Stress-induced anhedonia is associated with an increase in Alzheimer's disease-related markers. British Journal Pharmacological, 165 (4), 897-907.

Caspi, A., Vishne, T., Sasson, Y., Gross, R., Livne, A., y Zohar, J. (2008). Relationship between childhood sexual abuse and obsessive-compulsive disorder: case control study. Israel Journal of Psychiatry \& Related Sciences, 45 (3), 177-182.

Censo de población y vivienda 2010. Disponible en http://cuentame.inegi.org.mx/monografias/informacion/chis/ poblacion/

Del Giudice, M. (2014). Early stress and human behavioral development: emerging evolutionary perspectives. Journal of Developmental Origins of Health and Disease, 5 (4), 270-280.

Dosaev, T., Prakash, J., y Livshits, G. (2014). Contribution of body composition components and soft-tissue biochemical factors to genetic variation of body mass index (BMI) in an ethnically homogeneous population. American Journal of Human Biology, doi: 10.1002/ajhb.22583.

Elenkov, I. J., Papanicolaou, D. A., Wilder, R. L., y Chrousos, G. P. (1996). Modulatory effects of glucocorticoids and catecholamines on human interleukin-12 and interleukin-10 production: clinical implications. Proceedings of the Association of American Physicians, 108 (5), 374-381.

Elenkov, I. J., Webster, E. L., Torpy, D. J., y Chrousos, G. P. (1999). Stress, corticotropin-releasing hormone, glucocorticoids, and the immune/inflammatory response: acute and chronic effects. Annals of the New York Academy of Sciences, 876, 1-11.

Espinosa, G., Santos, E., Cervera, R., Piette, J. C., de la Red, G., Gil, V., Font, J., Couch, R., Ingelmo, M., y Asherson, R. A. (2003). Adrenal involvement in the antiphospholipid syndrome: clinical and immunologic characteristics of 86 patients. Medicine (Baltimore), 82 (2), 106-118.

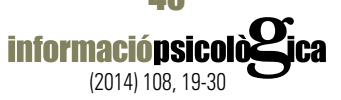


Estadística de nupcialidad; INEGI, 2012. Disponible en http://www3.inegi.org.mx/sistemas/mexicocifras/default. aspx?e=7

Ferro, M. A., y Boyle, M. H. (2014). The Impact of Chronic Physical Illness, Maternal Depressive Symptoms, Family Functioning, and Self-esteem on Symptoms of Anxiety and Depression in Children. Journal of Abnormal Child Psychology, PMID: 24938212.

Gibb, B. E., Chelminski, I., y Zimmerman, M. (2007). Childhood emotional, physical, and sexual abuse, and diagnoses of depressive and anxiety disorders in adult psychiatric outpatients. Depression Anxiety, 24 (4), 256-263.

Green, J. G., McLaughlin, K. A., Berglund, P. A., Gruber, M. J., Sampson N. A., Zaslavsky, A. M., y Kessler, R. C. (2010). Childhood adversities and adult psychiatric disorders in the national comorbidity survey replication I: associations with first onset of DSM-IV disorders. Archives of General Psychiatry, 67 (2), 113-123.

Höschl, C., y Hajek, T. (2001). Hippocampal damage mediated by corticosteroids-a neuropsychiatric research challenge. European Archives of Psychiatry and Clinical Neuroscience, 251, Suppl 2, II81-88.

Hovens, J. G., Wiersma, J. E., Giltay, E. J., van Oppen, P., Spinhoven, P., Penninx, B. W., y Zitman, F. G. (2010). Childhood life events and childhood trauma in adult patients with depressive, anxiety and comorbid disorders vs. controls. Acta Psychiatrica Scandinavica, 122 (1), 66-74.

Kessler, R. C., McLaughlin, K. A., Green, J. G., Gruber, M. J., Sampson, N. A., Zaslavsky, A. M., Aguilar-Gaxiola, S., Alhamzawi. A. O., Alonso, J., Angermeyer, M., Benjet, C., Bromet, E., Chatterji, S., de Girolamo, G., Demyttenaere, K., Fayyad, J., Florescu, S., Gal, G., Gureje, O., Haro, J. M., Hu, C. Y., Karam, E. G., Kawakami, N., Lee, S., Lépine, J. P., Ormel, J., Posada-Villa, J., Sagar, R., Tsang, A., Ustün, T. B., Vassilev, S., Viana, M. C., y Williams, D. R. (2010). Childhood adversities and adult psychopathology in the WHO World Mental Health Surveys. . The British Journal of Psychiatry, 197 (5), 378-385.

Khoury, L., Tang, Y. L., Bradley, B., Cubells, J. F., y Ressler, K. J. (2010). Substance use, childhood traumatic experience, and posttraumatic stress disorder in an urban civilian population. Depression Anxiety, 27 (12), 10771086.

Lambert, K., y Spinath, B. (2013). [Changes in psychological stress after interventions in children and adolescents with mathematical learning disabilities]. Z Kinder Jugendpsychiatr Psychother, 41 (1), 23-34.

Lang, A. J., Stein, M. B., Kennedy, C. M., y Foy, D. W. (2004). Adult psychopathology and intimate partner violence among survivors of childhood maltreatment. Journal Interpers Violence, 19 (10), 1102-118.

Leonard, B. E. (2006). HPA and immune axes in stress: involvement of the serotonergic system. Neuroimmunomodulation, 13 (5-6), 268-76.

Lightman, S. L. (2008). The neuroendocrinology of stress: a never ending story. Journal Neuroendocrinology, 20 (6), 880-884.

Loredo-Martínez, N., Mejía-Jiménez, D., Jiménez-Bautista N., y Matus-Miranda R. (2009). Nivel de estrés en niños(as) de primer año de primaria y correlación con alteraciones en su conducta. Revista Enfermería Universitaria ENEO-UNAM, 6, (4), 7-14.

Maganto-Mateo, C. (2004). Consecuencias psicopatologicas del divorcio en los hijos. Disponible en: http://www. sc.ehu.es/ptwmamac/Capi_libro/40c.pdfC.

Mäenpää, H., Savolainen, A., Lehto, M. U., y Belt, E. A. (2001). Multiple stress fractures in a young girl with chronic idiopathic arthritis. Extended case report. Joint Bone Spine, 68 (5), 438-442.

McLaughlin, K. A., Green, J. G, Gruber, M. J., Sampson, N. A., Zaslavsky, A. M., y Kessler, R. C. (2010). Childhood adversities and adult psychopathology in the National Comorbidity Survey Replication (NCS-R) III: associations with functional impairment related to DSM-IV disorders. Psychological Medicine, 40 (5), 847-859.

Molnar, B. E., Buka, S. L., y Kessler, R. C. (2001). Child sexual abuse and subsequent psychopathology: results from the National Comorbidity Survey. The American Journal of Public Health, 91 (5), 753-760.

Ojeda, N., y González- Fafoaga E. (2008). Divorcio y separación conyugal en México. Revista Mexicana de Sociología, 70 (1), 111-145.

Oros, L. B., y Vogel, G K. (2005). Eventos que generan estrés en la infancia: diferencias por sexo y edad. Enfoques, XVII (1), 85-101. 
Roy, A. (2002). Childhood trauma and neuroticism as an adult: possible implication for the development of the common psychiatric disorders and suicidal behaviour. Psychological Medicine, 32 (8), 1471-1474.

Sareen, J., Fleisher, W., Cox, B. J., Hassard, S., y Stein, M. B. (2005). Childhood adversity and perceived need for mental health care: findings from a Canadian community sample. Journal of Nervous And Mental Disease, 193 (6), 396-404.

Sävendahl L. (2012). The effect of acute and chronic stress on growth. Science Signaling, 5 (247) pt9.

Schmeer, K. K., y Kroeger, R. A. (2011). Union Type and Depressive Symptoms Among Mexican Adults. Journal of Family Issues, 32 (12), 1597-1621.

Secretaría de Salud. (2001). Programa de Acción en Salud Mental. SERSAME. México. Disponible en http://sersame. salud.gob.mx/pdf/pasm_intro.pdf

Straub, R. H., y Härle, P. (2005). [Stress, hormones, and neuronal signals in the pathophysiology of rheumatoid arthritis. The negative impact on chronic inflammation]. Medizinische Klinik (Munich), 100 (12), 794-803.

Talge, N. M., Neal, C., y Glover, V. (2007). Early Stress, Translational Research and Prevention Science Network: Fetal and Neonatal Experience on Child and Adolescent Mental Health. Antenatal maternal stress and longterm effects on child neurodevelopment: how and why? Journal of Child Psychology and Psychiatry, 48 (3-4), 245-261. Review.

Tasker, R. C., Roe, M. F., Bloxham, D. M., White, D. K., Ross-Russell, R. I., y O'Donnell, D. R. (2004). The neuroendocrine stress response and severity of acute respiratory syncytial virus bronchiolitis in infancy. Intensive Care Medicine, 30 (12), 2257-2262.

Tharandt, L., Rosanowski, C., Windeck, R., Benker, G., Hackenberg, K., y Reinwein, D. (1981). The metabolic serum clearance of GnRH. Relationship to age, sex and male puberty. Hormone and Metabolic Research, 13 (5), 277-282.

Turner-Cobb, J. M., Rixon, L., y Jessop, D. S. (2011). Hypothalamic-pituitary-adrenal axis activity and upper respiratory tract infection in young children transitioning to primary school. Psychopharmacology, 214 (1), 309-317.

Vega, C. Z., Espindola, S., Nava, C. y Soria, R. (2009). Estrés y ambiente familiar en niños. Revista PsicologiaCientifica.com, 11 (10). Disponible en: http://www.psicologiacientifica.com/estres-y-ambiente-familiar-ninos.

Vega, C. Z., González, F., Anguiano S., Nava, C., y Soria R. (2009). Habilidades sociales y estrés infantil. Revista de Conducta, Salud y Temas Sociales, 1 (1), 7-13.

Verduzco-Alvarez Icaza, M. A., Gómez, M. E. L., y Durán-Patiño, C. (2004). La influencia de la autoestima en la percepción del estrés y el afrontamiento en niños de edad escolar. Salud Mental, 27 (4), 18-25.

Villatoro, J.; Quiroz, N.; Gutiérrez M. L., Díaz, M., y Amador, N. (2006). ¿Cómo educamos a nuestros/as hijos/as? Encuesta de Maltrato Infantil y Factores Asociados 2006. Instituto Nacional de las Mujeres (INMUJERES). Instituto Nacional de Psiquiatría Ramón de la Fuente Muñiz (INPRFM).México, D. F. Disponible en: http://cedoc.inmujeres.gob.mx/documentos_download/100769.pdf

Villatoro-Velázquez, J. A., Medina-Mora, M. E., Fleiz-Bautista, C., Téllez-Rojo, M. M., Mendoza-Alvarado, L. R., RomeroMartínez, M., Gutiérrez-Reyes, J. P., Castro-Tinoco, M., Hernández-Ávila, M., Tena-Tamayo, C., Alvear Sevilla, C., y Guisa-Cruz V. (2011). Instituto Nacional de Psiquiatría Ramón de la Fuente Muñiz; Instituto Nacional de Salud Pública; Secretaría de Salud. Encuesta Nacional de Adicciones 2011: Reporte de Drogas. Disponible en: www.conadic.gob.mx, www.cenadic.salud.gob.mx, www.insp.mx

Wingenfeld, K., Schaffrath, C., Rullkoetter, N., Mensebach, C., Schlosser, N., Beblo. T., Driessen, M., y Meyer, B. (2011). Associations of childhood trauma, trauma in adulthood and previous-year stress with psychopathology in patients with major depression and borderline personality disorder. Child Abuse \& Neglect, 35 (8), 647-54.

Wolchik, S. A., Wilcox, K. L., Tein, J. Y., y Sandler, I. N. (2000). Maternal acceptance and consistency of discipline as buffers of divorce stressors on children's psychological adjustment problems. Journal of Abnormal Child Psychology, 28 (1), 87-102.

Wonderlich, S. A., Rosenfeldt, S., Crosby, R. D., Mitchell, J. E., Engel, S. G., Smyth, J., y Miltenberger, R. (2007). The effects of childhood trauma on daily mood lability and comorbid psychopathology in bulimia nervosa. Journal Trauma Stress, 20 (1), 77-87. 